Physics

Physics Research Publications

\title{
Origin of the large band-gap bowing in highly mismatched semiconductor alloys
}

J. Wu, W. Walukiewicz, K. M. Yu, J. W. Ager, E. E. Haller, I. Miotkowski, A. K. Ramdas, C. H. Su, I. K. Sou, R. C. C. Perera, and J. D. Denlinger 


\title{
Origin of the large band-gap bowing in highly mismatched semiconductor alloys
}

\author{
J. Wu, ${ }^{1,2}$ W. Walukiewicz, ${ }^{2, *}$ K. M. Yu, ${ }^{2}$ J. W. Ager III, ${ }^{2}$ E. E. Haller,,${ }^{2,3}$ I. Miotkowski, ${ }^{4}$ A. K. Ramdas, ${ }^{4}$ Ching-Hua Su, ${ }^{5}$ \\ I. K. Sou, ${ }^{6}$ R. C. C. Perera, ${ }^{7}$ and J. D. Denlinger ${ }^{8}$ \\ ${ }^{1}$ Applied Science and Technology Graduate Group, University of California, Berkeley, California 94720 \\ ${ }^{2}$ Materials Sciences Division, Lawrence Berkeley National Laboratory, Berkeley, California 94720 \\ ${ }^{3}$ Department of Materials Science and Engineering, University of California, Berkeley, California 94720 \\ ${ }^{4}$ Department of Physics, Purdue University, West Lafayette, Indiana 47907 \\ ${ }^{5}$ SD46 Science Directorate, NASA Marshall Space Flight Center, Huntsville, Alabama 35812 \\ ${ }^{6}$ Department of Physics, Hong Kong University of Science and Technology, Clear Water Bay, Kowloon, Hong Kong, China \\ ${ }^{7}$ Center for X-Ray Optics, Lawrence Berkeley National Laboratory, Berkeley, California 94720 \\ ${ }^{8}$ Advance Light Source, Lawrence Berkeley National Laboratory, Berkeley, California 94720
}

(Received 12 November 2002; published 28 January 2003)

\begin{abstract}
Photomodulated reflection, optical absorption, and photoluminescence spectroscopies have been used to measure the composition dependence of interband optical transitions in $\mathrm{ZnSe}_{1-x} \mathrm{Te}_{x}$ and $\mathrm{ZnS}_{1-x} \mathrm{Te}_{x}$ alloys. The results reveal entirely different origins of the large band-gap bowing for small and large Te content. On the Te-rich side, the reduction of the band gap is well explained by the band anticrossing interaction between the Se or S localized states and the ZnTe conduction-band states. On the Se- or S-rich side, an interaction between the localized Te states and the degenerate $\Gamma$ valence bands of $\mathrm{ZnSe}$ or $\mathrm{ZnS}$ is responsible for the band-gap reduction and the rapid increase of the spin-orbit splitting with increasing Te concentration. Results of the soft-x-ray emission experiment provide direct proof of the valence-band anticrossing interaction. The band-gap bowing in the entire composition range is accounted for by a linear interpolation between the conduction-band anticrossing and valence-band anticrossing models.
\end{abstract}

DOI: 10.1103/PhysRevB.67.035207

PACS number(s): 78.40.-q, 74.70.Dd, 78.55.Et

\section{INTRODUCTION}

Despite decades of extensive studies, there is no commonly accepted explanation of the origin of the widely observed, nonlinear dependence of the fundamental band gap on the composition of semiconductor alloys. Typically, it has been assumed that the deviation from a linear dependence predicted by the virtual crystal approximation ${ }^{1}$ (VCA) can be accounted for by including effects of disorder on the conduction- and valence-band edge. In this approach, the composition dependence of the band gap is described by a second-order polynomial with the quadratic term proportional to the so-called bowing parameter. In a more recent treatment of this issue, it was argued that the bowing parameter can be decomposed into three parts originating from charge exchange, volume deformation, and structural relaxation, respectively. ${ }^{2}$ But in this theory it was also assumed that a single bowing parameter can describe the band-gap behavior in the whole composition range.

Recent progress in the epitaxial growth techniques has led to successful synthesis of alloys of distinctly different semiconductor materials. The most prominent class of such highly mismatched alloys (HMA's) is $\operatorname{IIIV}_{1-x} \mathrm{~N}_{x}$ materials, in which standard group-III-V compounds are alloyed with group-III nitrides. The $\mathrm{N}$-induced band-gap reduction and the greatly reduced pressure dependence of the band gap in these alloys significantly deviate from the VCA-based predictions. It has been demonstrated that the composition dependence of the band gap of these alloys cannot be described using a single bowing parameter. A band-anticrossing (BAC) model $^{3,4}$ has been developed to explain these unusual effects. In the BAC model, the anticrossing interaction between the previously observed $\mathrm{N}$ resonant states and the conductionband states of the host semiconductor modifies the electronic structure of the material and is responsible for these observations. The model has not only explained the observed experimental results but also predicted other effects in IIIV $_{1-x} \mathrm{~N}_{x}$ alloys that has been now experimentally confirmed. ${ }^{5,6}$

Recently, the BAC model has also been applied to Te-rich $\mathrm{ZnSe}_{1-x} \mathrm{Te}_{x}$ and $\mathrm{ZnS}_{1-x} \mathrm{Te}_{x}$ alloys ( $\left.x \geq 0.8\right)$, in which strong reductions in the band gap and in the pressure coefficients similar to those in IIIV $_{1-x} \mathrm{~N}_{x}$ have also been observed. ${ }^{7}$ These observations have been fully accounted for by the anticrossing interaction between the $\mathrm{S}$ or Se localized levels and the $\mathrm{ZnTe}$ conduction-band edge. In its current form, the BAC model is applicable to the cases of dilute alloys, where a small fraction of highly electronegative atoms replace more metallic anions. In this article, we report our studies of the electronic structure of $\mathrm{ZnSe}_{1-x} \mathrm{Te}_{x}$ and $\mathrm{ZnS}_{1-x} \mathrm{Te}_{x}$ alloys in the entire composition range. The combination of optical and soft-x-ray emission experiments shows the complex nature of the band-gap bowing in these materials. The results demonstrate that on the Se-rich (or S-rich) side, the band-gap bowing is mostly determined by an anticrossing interaction between the Te localized level and the extended states of the $\mathrm{ZnSe}$ (or ZnS) valence bands near the Brillouin-zone center.

\section{EXPERIMENT}

Bulk $\mathrm{ZnSe}_{1-x} \mathrm{Te}_{x}$ crystals were grown by either a modified Bridgman method ${ }^{8}$ on the Te-rich side, or by the physical vapor transport method ${ }^{9}$ on the Se-rich side. Thin films of $\mathrm{ZnS}_{1-x} \mathrm{Te}_{x}$ were grown by the molecular beam epitaxy technique in a VG V80H system on a semi-insulating (001) GaAs substrate. ${ }^{10}$ The thickness ranged from 0.6 to $3.1 \mu \mathrm{m}$. 


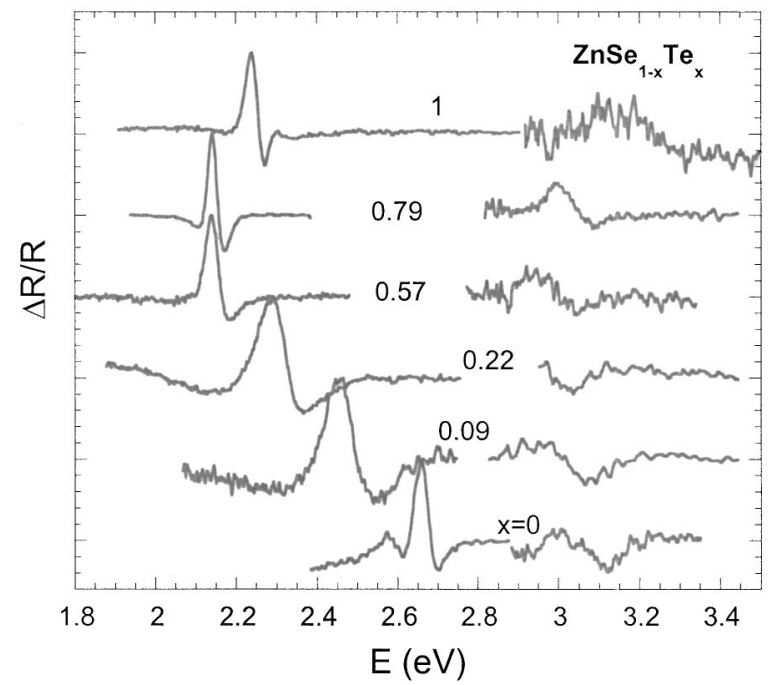

FIG. 1. Typical PR spectrum obtained at room temperature for $\mathrm{ZnSe}_{1-x} \mathrm{Te}_{x}$ alloys with a range of $x$.

The energies of the critical point transitions were measured using the photomodulated reflectance (PR) technique at room temperature. Quasimonochromatic light from a halogen tungsten lamp dispersed by a $0.5-\mathrm{m}$ monochromator was focused on the sample as a probe beam. A chopped $\mathrm{HeCd}$ laser beam provided the modulation. The transition energies and the linewidth broadenings were determined by fitting the PR spectra to the Aspnes third-derivative functional form. ${ }^{11}$ Photoluminescence (PL) signals were generated in the backscattering geometry by excitation with the $476.5-\mathrm{nm}$ line of an argon laser and were dispersed by a 1-m double-grating monochromator.

The soft-x-ray fluorescence data of $\mathrm{ZnS}_{1-x} \mathrm{Te}_{x}$ were obtained by using soft-x-ray absorption and soft-x-ray emission spectroscopies at beamline 8.0 of the Advanced Light Source. The combination of these two techniques enables us to derive information about the partial density of states of the conduction band and valence band for a large range of composition. $^{12}$

\section{RESULTS AND DISCUSSION}

\section{A. Valence-band anticrossing}

Figure 1 shows typical PR spectra obtained from the $\mathrm{ZnSe}_{1-x} \mathrm{Te}_{x}$ alloys with several compositions. The feature at lower energy $\left(E_{g}\right)$ corresponds to the critical transition from the top of the degenerate heavy-hole (hh) and light-hole (lh) valence bands to the conduction-band edge. The feature at higher energy $\left(E_{g}+\Delta_{0}\right)$ is attributed to the transition from the top of the spin-orbit (s.o.) valence-band to the conduction-band edge.

The composition dependencies of the fundamental band gap $\left(E_{g}\right)$ and the spin-orbit splitting $\left(\Delta_{0}\right)$ are shown in Fig. 2. The data shown in Fig. 2 are in quantitative agreement with previous observations by electroreflectance spectroscopy ${ }^{13}$ and photoconductivity measurements. ${ }^{14}$ The energy gap shows a convex dependence on the Te concentration that deviates significantly from the linear dependence as

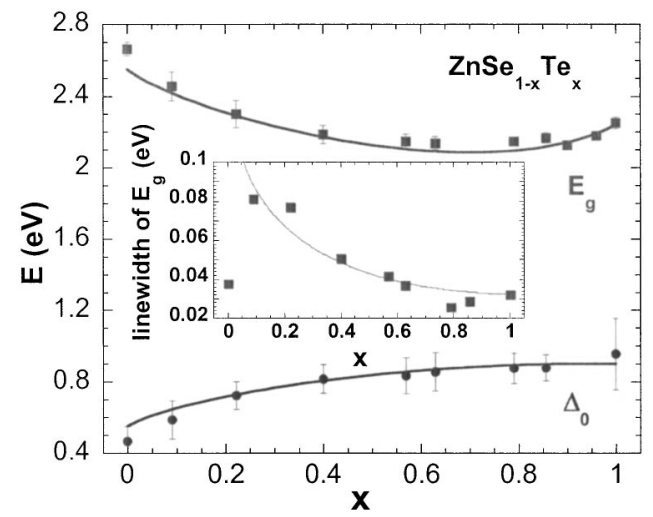

FIG. 2. $E_{g}$ and $\Delta_{0}$ of $\mathrm{ZnSe}_{1-x} \mathrm{Te}_{x}$ as a function of $x$ measured by photomodulated reflectance. The curves show the calculated values. The inset shows the composition dependence of the linewidth of the $E_{g}$ transition. The curve is a fit based on the BAC model.

predicted by the VCA. The spin-orbit splitting, in contrast, shows a concave dependence on the alloy composition. $\Delta_{0}$ increases rapidly for small $x$ until $x \sim 0.5$. The different behavior of $\Delta_{0}$ on the Se-rich side and the Te-rich side suggests different origins of the band-gap bowing in Se- and Te-rich $\mathrm{ZnSe}_{1-x} \mathrm{Te}_{x}$ alloys.

In Te-rich alloys $(x \rightarrow 1)$, the BAC model predicts a new conduction-band edge formed by the anticrossing interaction between the Se localized states and the $\Gamma$ conduction band of $\mathrm{ZnTe}^{7}$ The localized Se level lies at $2.85 \mathrm{eV}$ above the valence-band edge of $\mathrm{ZnTe}$. It has been shown ${ }^{7}$ that the perturbation of the Se localized states on the ZnTe conduction band successfully explains the composition and hydrostatic pressure dependencies of the fundamental band gap on the Te-rich side $\left(E_{g}^{\text {Te rich }}\right)$.

For Se-rich alloys $(x \rightarrow 0)$, the rapid increase in $\Delta_{0}$ at small $x$ implies a perturbation on the valence bands introduced by the incorporation of $\mathrm{ZnTe}$ in $\mathrm{ZnSe}$. It has been recognized very early ${ }^{15}$ that the isoelectronic Te impurity substituting $\mathrm{Se}$ in $\mathrm{ZnSe}$ forms a localized level above the valence-band edge. At dilute concentrations, the Te level gives rise to a strong broad luminescence line below the band gap. ${ }^{15}$ In close analogy to the anticrossing effect between the Se localized level and the ZnTe conduction band on the Te-rich side, ${ }^{7}$ we consider the hybridization between the localized Te level and the valence bands of the host material. The original four $\Gamma_{8}$ and two $\Gamma_{7}$ valence bands are described by the conventional $6 \times 6$ Kohn-Luttinger $\mathbf{k} \cdot \mathbf{p}$ matrix ${ }^{16}$ with band-edge energies set at corresponding VCA values. An $8 \times 8$ Hamiltonian matrix $\left(H_{v}\right)$ is formed by augmenting the $6 \times 6$ matrix with two localized Te states with energy $E_{\mathrm{Te}}$ and opposite spins. The valence-band restructuring is computed by diagonalizing this $8 \times 8$ matrix. Choosing as a basis the six time-reversal symmetry-invariant wave functions ${ }^{16}$ for the valence bands, the matrix elements for the columns corresponding to the Te states are listed in Table I.

In Table I, $V$ is the hybridization energy between the Te localized states and the three wave functions of the $\Gamma_{4}$ representation of the $T_{d}$ crystal group,

$$
V \equiv\langle\mathrm{Te}|U| X\rangle=\langle\mathrm{Te}|U| Y\rangle=\langle\mathrm{Te}|U| Z\rangle=C_{\mathrm{ZnSe}-\mathrm{Te}} \sqrt{x} .
$$


TABLE I. The matrix elements of $H_{v}$ involving the Te localized level.

\begin{tabular}{|c|c|c|c|c|c|c|c|c|}
\hline & $\left|\frac{3}{2}, \frac{3}{2}\right\rangle$ & $\left|\frac{3}{2}, \frac{1}{2}\right\rangle$ & $\left|\frac{3}{2},-\frac{1}{2}\right\rangle$ & $\left|\frac{3}{2},-\frac{3}{2}\right\rangle$ & $\left|\frac{1}{2}, \frac{1}{2}\right\rangle$ & $\left|\frac{1}{2},-\frac{1}{2}\right\rangle$ & $|\mathrm{Te} \uparrow\rangle$ & $|\mathrm{Te} \downarrow\rangle$ \\
\hline$|\mathrm{Te} \uparrow\rangle$ & $\frac{(1+i)}{\sqrt{2}} V$ & $-\frac{i \sqrt{2}}{\sqrt{3}} V$ & $\frac{(1-i)}{\sqrt{6}} V$ & 0 & $\frac{1}{\sqrt{3}} V$ & $-\frac{(1+i)}{\sqrt{3}} V$ & $E_{\mathrm{Te}}$ & 0 \\
\hline$|\mathrm{Te} \downarrow\rangle$ & 0 & $\frac{(-1+i)}{\sqrt{6}} V$ & $\frac{\sqrt{2}}{\sqrt{3}} V$ & $\frac{(1+i)}{\sqrt{2}} V$ & $\frac{(1+i)}{\sqrt{3}} V$ & $\frac{i}{\sqrt{3}} V$ & 0 & $E_{\mathrm{Te}}$ \\
\hline
\end{tabular}

In Eq. (1), we assume a square-root dependence on the impurity concentration similar to that obtained in the case of the conduction-band hybridization. ${ }^{4,7} C_{\mathrm{ZnSe}-\mathrm{Te}}$ is a parameter that describes the coupling strength and is to be determined by fitting with experimental data. The four doubly degenerate eigenvalues of $H_{v}$ have been computed. In the Brillouinzone center, they can be named according to the nature of their wave functions as $E_{\text {Te-like }}, E_{\text {hh-like }}, E_{\text {lh-like }}$, and $E_{\text {s.o.like }}$ in order of descending eigenenergy. Since the energy level of the Te localized states is located above the top of the original valence band, a new edge of valence band is formed at energy $E_{\text {Te-like }}$. The fundamental band gap in Se-rich $\mathrm{ZnSe}_{1-x} \mathrm{Te}_{x}$, denoted as $E_{g}^{\text {Se-rich }}(x)$, is attributed to the transition between the VCA conduction-band edge and this newly formed valence-band edge. The spin-orbit splitting is given by the energy separation between $E_{\text {Te-like }}$ and $E_{\text {s.o.-like }}$. We note that the orthogonality between opposite spin states leads to two zero-valued off-diagonal matrix elements in Table I, i.e., $\left\langle\mathrm{Te} \downarrow \mid \frac{3}{2}, \frac{3}{2}\right\rangle$ and $\left\langle\mathrm{Te} \uparrow \mid \frac{3}{2},-\frac{3}{2}\right\rangle$. As a result, the heavy-hole band does not mix with the Te states at the $\Gamma$ point, and the energy of the heavy-hole band edge remains unchanged. The light-hole and spin-orbit valence-band edges, on the other hand, are pushed downwards by the anticrossing repulsion from the higher-lying Te level. The energy degeneracy between the heavy-hole and the light-hole bands in the Brillouin-zone center is thus removed. This anticrossing interaction not only shifts the band edge in the Brillouin-zone center but also affects the dispersion relations of the valence bands. Calculations show that the restructured valence bands become largely nonparabolic, similar to the case in the conduction-band anticrossing. 5,6

In order to fit the experimental data in the entire composition range $(0 \leqslant x \leqslant 1)$, we adopt a linear interpolation scheme, similar to the spirit of the original VCA interpolation, between these two effects, i.e., the conduction-band $\mathrm{BAC}$ on the Te-rich side and the valence-band mixing on the Se-rich side. For the composition dependence of the fundamental band gap, it is written as

$$
E_{g}(x)=(1-x) E_{g}^{\mathrm{Se}-\text { rich }}(x)+x E_{g}^{\mathrm{Te}-\text { rich }}(x) .
$$

The linear interpolation weights the importance of these two effects by the concentration of the majority component from each side.

The band gap and the spin-orbit splitting obtained from Fig. 1 are shown in Fig. 2 in comparison with the calculations. The best agreement with experiments can be obtained by setting the coupling parameter as $C_{\mathrm{ZnSe}-\mathrm{Te}}=0.30 \mathrm{eV}$ and the Te localized level at $0.10 \mathrm{eV}$ above the top of the original $\mathrm{ZnSe}$ valence band. This energy location is close to the value reported in Ref. 15 for the Te level in low-Te-concentration ZnSeTe alloys.

We note the unusual composition dependence of the linewidth of the $E_{g}$ feature shown in Fig. 1. An abrupt broadening of the linewidth is observed on the Se-rich side. The linewidth as a function of composition is shown in the inset of Fig. 2. This composition dependence of the PR linewidth is quite similar to the behavior of the previously reported PL linewidth in $\mathrm{ZnSe}_{1-x} \mathrm{Te}_{x}$ alloys. ${ }^{14}$ The asymmetry in the PR line-broadening behavior is associated with a change in the nature of the band-edge states. It has been found by a Green's function calculation ${ }^{17}$ that the energy broadening of an eigenstate in the localized-extended hybridization system is proportional to the admixture of the localized states in its wave function. The curve in the inset is a fit to the linewidth data based on the percentage of localized states in the wave function of $E_{\mathrm{Te} \text {-like }}$. The large linewidth for small $x$ values is associated with the localized nature of the Te-like states at the top of the valence band. For $x$ close to 1, the delocalized nature of both the conduction- and the valence-band edges results in a narrow linewidth for the PR spectra.

\section{B. Soft-x-ray emission experiments}

It is important to note that according to this model, the main contribution to the band-gap bowing originates from the downward shift of the conduction-band edge, on the Terich side, and an upward shift of the valence band, on the Se-rich side. To independently confirm this assertion, one needs to determine the conduction- and valence-band offsets as a function of composition. In this context, we have used soft-x-ray emission (SXE) spectroscopy to measure the valence-band shift in $\mathrm{ZnS}_{1-x} \mathrm{Te}_{x}$. A large shift of the valence band is expected in this alloy system, as it has been known for a long time that the localized Te level is separated by as much as $1.2 \mathrm{eV}$ from the valence-band edge of $\mathrm{ZnS} .^{10}$

Figure 3(a) shows typical SXE spectra for a few $\mathrm{ZnS}_{1-x} \mathrm{Te}_{x}$ samples excited at the $\mathrm{S} 2 p$ level. In this experiment, the excitation $\mathrm{x}$-ray energy first scanned across the $\mathrm{S}$ $2 p$ core levels, and the partial fluorescence yield was recorded as the x-ray absorption spectrum. The threshold energy of the excitation determines the energy of the conduction-band minimum with respect to the core level. They are shown as the right-hand side of the curves in Fig. $3(\mathrm{a})$. The doublet structure due to the $\mathrm{S} 2 p_{3 / 2}$ and $\mathrm{S} 2 p_{1 / 2}$ splitting $(\sim 1.6 \mathrm{eV})$ is clearly resolved, ${ }^{18}$ especially on the $\mathrm{ZnTe}$-rich side. The excitation energy was then tuned to the 

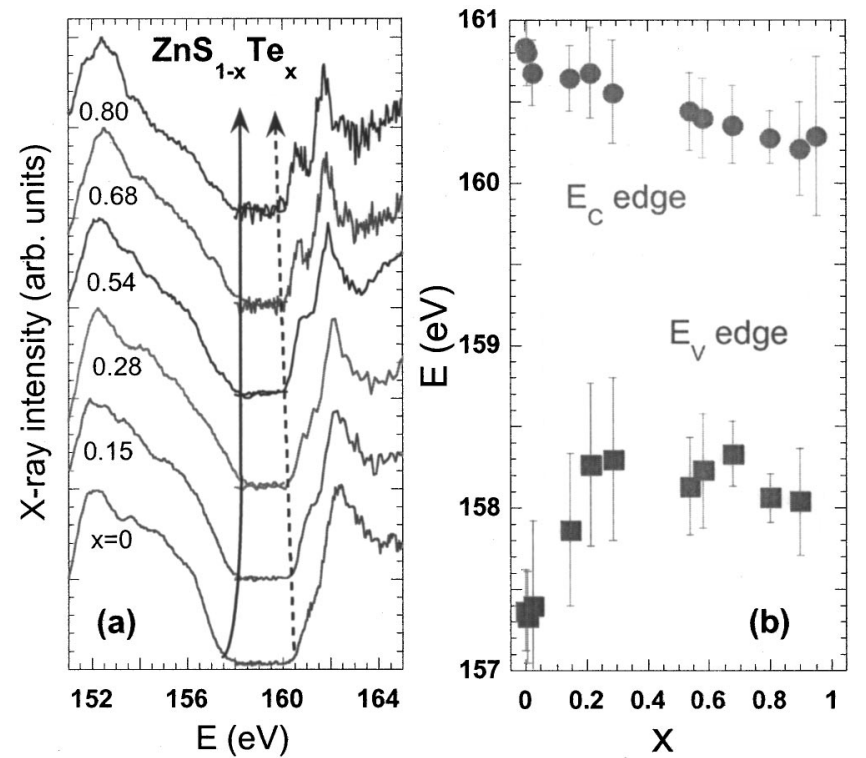

FIG. 3. (a) Typical S $2 p$ soft-x-ray emission and absorption spectrum of $\mathrm{ZnS}_{1-x} \mathrm{Te}_{x}$ alloys. (b) Conduction-band minimum and valence-band maximum determined by soft-x-ray emission as a function of $x$.

edge of the conduction band so that only the $\mathrm{S} 2 p_{3 / 2}$ core level was ionized. The $\mathrm{x}$-ray emission due to electronic transitions from the upper valence-band region to this core level was monitored with an energy-dispersive detector. The emission spectrum is shown in Fig. 3(a) as the left-hand side of the SXE curves. In this way the relative shifts of the conduction-band minimum [schematically shown by the dashed arrow in Fig. 3(a)] and valence-band maximum [solid arrow in Fig. 3(a)] as a function of composition are directly measured. The results are summarized in Fig. 3(b). It is evident that, in agreement with the BAC model, the top of the valence band exhibits a strongly nonlinear composition dependence on the S-rich side. This upward shift is caused by the transition in the nature of the highest valence-band edge from that of extended states of pure $\mathrm{ZnS}$ to localized Te-like states hybridized with the extended valence-band states of the $\mathrm{ZnS}$ matrix.

Figure 4 summarizes the transition energies measured by different techniques over the entire composition range of the $\mathrm{ZnS}_{1-x} \mathrm{Te}_{x}$ alloy. The data points from PR and absorption experiments that measure the large density of extended states show a strong band-gap bowing. On the other hand, the PL peak is shifted to lower energies as it is associated with optical transitions to deep states. The energy gap measured by SXE in Fig. 3 is also plotted in Fig. 4. Within experimental error, these gap energies agree with the band gaps measured by absorption PR experiments. This agreement confirms that the band edges measured by SXE in Fig. 3 are indeed the ones involved in the interband transitions observed in absorption and PR experiments.

Based on these observations, we have applied the bandanticrossing model to $\mathrm{ZnS}_{1-x} \mathrm{Te}_{x}$, too. The results are shown as curves in Fig. 4. On the Te-rich side, as shown in Ref. 7, an anticrossing interaction between the $\mathrm{ZnTe}$ conduction-

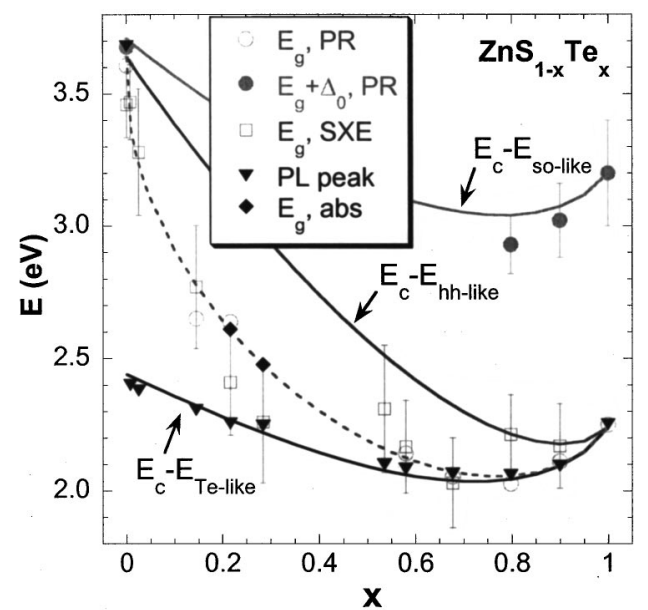

FIG. 4. PL peak energy and band gap measured by SXE, PR, and absorption experiments plotted as a function of $x$. The solid lines show the calculated energy difference between $E_{c}$ and $E_{\text {Te-like }}$, $E_{\text {hh-like }}$, and $E_{\text {s.o.-like }}$, respectively. The dashed line is an empirical interpolation between the $E_{c}-E_{\mathrm{Te}-\text { like }}$ and $E_{c}-E_{\mathrm{hh} \text {-like }}$ fitting to the composition dependence of the measured band gaps.

band minimum and the $\mathrm{S}$ level located at $\sim 0.36 \mathrm{eV}$ above the minimum was assumed. A valence-band anticrossing on the S-rich side was calculated and an interpolation as expressed in Eq. (2) was carried out over the whole composition range. The best fit is obtained by setting the Te level at $1.2 \mathrm{eV}$ above the valence-band maximum of $\mathrm{ZnS}$ and a coupling constant of $C_{\mathrm{ZnS}-\mathrm{Te}}=0.5 \mathrm{eV}$. It can be seen that the PL peak energy is predicted well by the transition from the conduction-band minimum to the highest, Te-like valenceband edge. The dashed line in Fig. 4 is an empirical weighted interpolation between calculated $E_{c}-E_{\mathrm{Te} \text {-like }}$ and $E_{c}$ $-E_{\mathrm{hh}-\mathrm{like}}$. The measured band gap of $\mathrm{ZnS}_{1-x} \mathrm{Te}_{x}$ deviates from $E_{c}-E_{\mathrm{hh}-\text { like }}$ as a result of the hybridization. But unlike in $\mathrm{ZnSe}_{1-x} \mathrm{Te}_{x}$, it does not immediately follow $E_{c}-E_{\mathrm{Te}-\text { like }}$ because of the much larger energy separation between the Te level and the valence-band maximum of $\mathrm{ZnS}$. Consequently, the fraction of extended states at the top of the restructured valence band (i.e., $E_{\mathrm{Te}-\mathrm{like}}$ ) is too small to contribute to the absorption or PR transitions. Such transitions are only observable when the anticrossing interaction becomes strong enough at higher Te concentrations.

The new understanding of the origin of the large band-gap bowing in these alloys also allows one to calculate the composition dependence of their conduction- and valence-band offsets. For example, similar to the expression of the band gap as a function of $x$ in Eq. (2), the conduction-band minimum $\left(E_{C}\right)$ of $\mathrm{ZnSe}_{1-x} \mathrm{Te}_{x}$ alloys can be written as

$$
E_{C}(x)=(1-x) E_{C}^{\mathrm{Se}-\text {-rich }}(x)+x E_{C}^{\mathrm{Te}-\text { rich }}(x),
$$

where $E_{C}^{\text {Se-rich }}(x)$ and $E_{C}^{\text {Te-rich }}(x)$ are the conduction-band edge on the Se-rich side (given by the VCA) and on the Te-rich side (given by the conduction BAC), respectively. The determination of the band offsets in the BAC model 
provides useful information for the design of heterostructure devices based on these alloys.

The calculations and their comparison with experimental results support the notion of different origins of the band-gap bowing in Se-rich (or S-rich) and Te-rich $\mathrm{ZnSe}_{1-x} \mathrm{Te}_{x}$ (or $\mathrm{ZnS}_{1-x} \mathrm{Te}_{x}$ ) alloys. The results are also important for understanding the doping behavior of these alloys. As has been shown recently, ${ }^{6}$ the $\mathrm{N}$-induced modification on the conduction-band structure of GaAs greatly improves the activation efficiency of shallow donors in $\mathrm{GaAs}_{1-x} \mathrm{~N}_{x}$ alloys. In the view of the findings in these II-VI alloys, we also expect that the downward shift of the conduction-band edge in Te-rich alloys and the upward shift in Se-rich (or S-rich) alloys should also lead to improvements in the activation efficiency of donors in the former and acceptors in the latter. These predictions are in agreement with experiments that have shown that alloying of $\mathrm{ZnSe}$ with small amounts of ZnTe greatly improves the activation efficiency of nitrogen acceptors. ${ }^{19}$ The enhancement of the hole effective mass as a result of the valence-band restructuring may also have advantageous effects in hole-mediated ferromagnetic semiconductors. ${ }^{20}$

\section{CONCLUSIONS}

In summary, we have used a variety of experimental techniques to determine the composition dependence of different band-edge energies in $\mathrm{ZnSe}_{1-x} \mathrm{Te}_{x}$ and $\mathrm{ZnS}_{1-x} \mathrm{Te}_{x}$. We show that the band-gap dependencies cannot be described in terms of constant bowing parameters. The composition dependences of the fundamental gaps and spin-orbit splitting are determined by anticrossing interactions between localized Se, S, and Te states and the extended band states. The model is general and applicable to all other highly mismatched alloys including $\mathrm{GaAs}_{1-x} \mathrm{Sb}_{x}$ and $\mathrm{GaP}_{1-x} \mathrm{Sb}_{x}$.

\section{ACKNOWLEDGMENTS}

This work is supported by the Director, Office of Science, Office of Basic Energy Sciences, Division of Materials Sciences and Engineering, of the U.S. Department of Energy under Contract No. DE-AC03-76SF00098. The work at Purdue received support from the National Science Foundation Grant No. DMR-0102699 and ECS-0129853 (SGER) as well as Purdue University Reinvestment Program. J. Wu acknowledges support from US NSF Grant No. DMR-0109844.
*Electronic mail: w_walukiewicz@lbl.gov

${ }^{1}$ J. A. VanVechten and T. K. Bergstresser, Phys. Rev. B 1, 3351 (1970); R. Hill and D. Richardson, J. Phys. C 4, L289 (1971).

${ }^{2}$ J. E. Bernard and A. Zunger, Phys. Rev. B 36, 3199 (1987).

${ }^{3}$ W. Shan, W. Walukiewicz, J. W. Ager III, E. E. Haller, J. F. Geisz, D. J. Friedman, J. M. Olson, and S. R. Kurz, Phys. Rev. Lett. 82, 1221 (1999).

${ }^{4}$ W. Walukiewicz, W. Shan, J. W. Ager III, D. R. Chamberlin, E. E. Hailer, J. F. Geisz, D. J. Friedman, J. M. Olson, and S. R. Kurtz, in Proceedings of the 195th Meeting of the Electrochemical Society, Seattle, WA, 1999, edited by V. K. Kapur, R. D. McConnell, D. Carlson, G. P. Caesar, and A. Rohatgi, Vol. 99-11 (The Electrochemical Society, Inc., Pennington, NJ, 1999), p. 190.

${ }^{5}$ C. Skierbiszewski, P. Perlin, P. Wiśniewski, W. Knap, T. Suski, W. Walukiewicz, W. Shan, J. W. Ager, E. E. Haller, J. F. Geisz, D. J. Friedman, J. M. Olson, and S. R. Kurtz, Appl. Phys. Lett. 76, 2409 (2000).

${ }^{6}$ K. M. Yu, W. Walukiewicz, W. Shan, J. W. Ager III, J. Wu, E. E. Haller, J. F. Geisz, D. J. Friedman, and J. M. Olson, Phys. Rev. B 61, R13 337 (2000).

${ }^{7}$ W. Walukiewicz, W. Shan, K. M. Yu, J. W. Auger III, E. E. Haller, I. Miotkowski, M. J. Seong, H. Alawadhi, and A. K. Ramdas, Phys. Rev. Lett. 85, 1552 (2000).

${ }^{8}$ M. J. Seong, H. Alawadhi, I. Miotkowski, A. K. Ramdas, and S. Miotkowska, Phys. Rev. B 60, R16 275 (1999).
${ }^{9}$ C. H. Su, S. Feth, S. Zhu, S. L. Lehoczky, and L. J. Wang, J. Appl. Phys. 88, 5148 (2000).

${ }^{10}$ I. K. Sou, K. S. Wong, Z. Y. Yang, H. Wang, and G. K. L. Wong, Appl. Phys. Lett. 65, 1915 (1995).

${ }^{11}$ D. E. Aspnes, Surf. Sci. 37, 418 (1973).

${ }^{12}$ C. B. Stagarescu, L. C. Duda, K. E. Smith, J. H. Guo, J. Nodgren, R. Singh, and T. D. Moustakas, Phys. Rev. B 54, R17 335 (1996).

${ }^{13}$ A. Ebina, Y. Sato, and T. Takahashi, Phys. Rev. Lett. 32, 1366 (1974).

${ }^{14}$ M. J. S. P. Brasil, R. E. Nahory, F. S. Turco-Sandroff, H. L. Gilchrist, and R. J. Martin, Appl. Phys. Lett. 58, 2509 (1991).

${ }^{15}$ D. Lee, A. Mysyrowicz, A. V. Nurmikko, and B. J. Fitzpatrick, Phys. Rev. Lett. 58, 1475 (1987).

${ }^{16}$ R. People and S. K. Sputz, Phys. Rev. B 41, 8431 (1990).

${ }^{17}$ J. Wu, W. Walukiewicz, and E. E. Haller, Phys. Rev. B 65, 233210 (2002).

${ }^{18}$ C. Heske, D. Eich, R. Fink, E. Umbach, T. van Buuren, C. Bostedt, L. J. Terminello, S. Kakar, M. M. Grush, T. A. Callcott, F. J. Himpsel, D. L. Ederer, R. C. C. Perera, W. Riedl, and F. Karg, Appl. Phys. Lett. 74, 1451 (1999).

${ }^{19}$ H. D. Jung, C. D. Song, S. Q. Wang, K. Arai, Z. Zhu, T. Yao, and H. Katayama-Yoshida, Appl. Phys. Lett. 70, 1143 (1997).

${ }^{20}$ T. Dietl, H. Ohno, and F. Matsukura, Phys. Rev. B 63, 195205 (2001). 